\title{
Information Technology for Assisted Living at Home: building a wireless infrastructure for assisted living
}

\author{
J. Mikael Eklund, Thomas Riisgaard Hansen, Jonathan Sprinkle and Shankar Sastry
}

\begin{abstract}
A heterogeneous wireless network to support a Home Health System is presented. This system integrates a set of smart sensors which are designed to provide health and security to the elder citizen living at home. The system facilitates privacy by performing local computation, it supports heterogeneous devices and it provide a platform and initial architecture for exploring the use of sensors with elderly people in the Information Technology for Assisted Living and Home project. The goal of this project is to provide alerts to care givers in the event of an accident or acute illness, and enable remote monitoring by authorized and authenticated care givers.
\end{abstract}

\section{INTRODUCTION}

The use of information and computer technology (ICT) for health care in the home is an area of rapidly expanding research and development that promises to provide improved care and quality of life while reducing the cost of providing this care. The Information Technology for Assisted Living at Home (ITALH) project enters this arena by allowing increased self-sufficiency and reduced direct care for the elderly and ill who may otherwise be forced to relocate from their own home to a group care facility.

The cost of moving to a group care facility is very high, both in terms of the monetary cost to the individual and their family or the social welfare system paying it, and very often to the health and happiness of the person who is required to leave their own home.

Most efforts to-date of applying ICT to health care in the home have focused on telemedicine, in which ICT is used to connect the patient and his/her biometric information to a health care provider either in real-time or into storage for analysis at a later time [1], [2], [3], [4]. While such functionality will be included in ITALH, the main objective in this project is to provide smart monitors and sensors that will alert the user and/or their care provider (be that a doctor, nurse, family member, friend, etc.) of events such as accidents or acute illness and to diagnostic events that could indicate a deterioration in condition. This differs from efforts such as [5], in which IR motions sensors are used to detect such events as accidental falls, in that the sensors themselves detect the event, not a central system. This reduces wireless bandwidth and greatly improves the privacy of the system by not streaming data constantly.

This work was supported by the Center for Information Technology Research in the Interest of Society (CITRIS)

J. Mikael Eklund, Jonathan Sprinkle and Shankar Sastry are with the Department of Electrical Engineering and Computer Sciences, University of California, Berkeley, CA, 94720 \{eklund, sprinkle, sastry\}@eecs.berkeley.edu

Thomas Riisgaard Hansen is with the Department of Computer Science, University of Aarhus, Aarhus, Denmark thomasr@daimi.au.dk
This paper introduces the wireless architecture, SensorNet, that forms the backbone of ITALH and presents the system in terms of its connectivity, modularity and security. SensorNet is a heterogeneous wireless network that connects sensor devices within the home and sensors worn by the user to a central Home Health System gateway and/or a mobile Gateway. The sensors themselves have embedded processing capability and are required to transmit only occasional information about their own status and messages notifying the central system when they detect a significant event. SensorNet supports Bluetooth and Zigbee (802.15.4) wireless as well as hard-wired connectivity via USB. The central system and the mobile gateway connect this network to the outside world via secure Internet and telephone service so that alerts can be sent out, and authorized care givers can have access to the system to check up on the user. Privacy and security are fundamental concerns build into ITALH and SensorNet.

\section{ApPlicAtion SCEnARIO}

SensorNet is an enabling component of the ITALH system. The application for this scenario is to provide assisted living to people requiring home care and to reduce the cost of providing this care while improving the safety and well-being of the user. The first setting for this system will be for the elderly, however it is expected to find use by many other citizens. To achieve this ITALH includes biometric, motion and environmental sensors which have embedded processing capabilities that allow them to perform analysis on the information and send alerts to the user and, if necessary, to care-givers, health care providers or emergency services.

A first prototype scenario is being developed in which each user will have a Home Health System that is able to monitor his or her health and activity in order to detect incidents and acute illness. The data is gathered from sensors worn by the user and by sensors mounted in the home. The alert signals are transmitted by the wireless SensorNet to the Home Health System. A mobile gateway is also included in SensorNet to allow for continuous monitoring by, for example, fall-sensors and bio-monitors when the user is away from home, and can provide a back-up to the home system. The home and mobile gateways receive the alert signals from the sensors and decide what the appropriate action to take will be. Options include querying the user to check on their status, storing (or forwarding) data on the event for future analysis by a health care provider, placing a telephone call to a neighbor, relative or health care service, or other options. Fig. 1 shows a schematic overview of the system. 


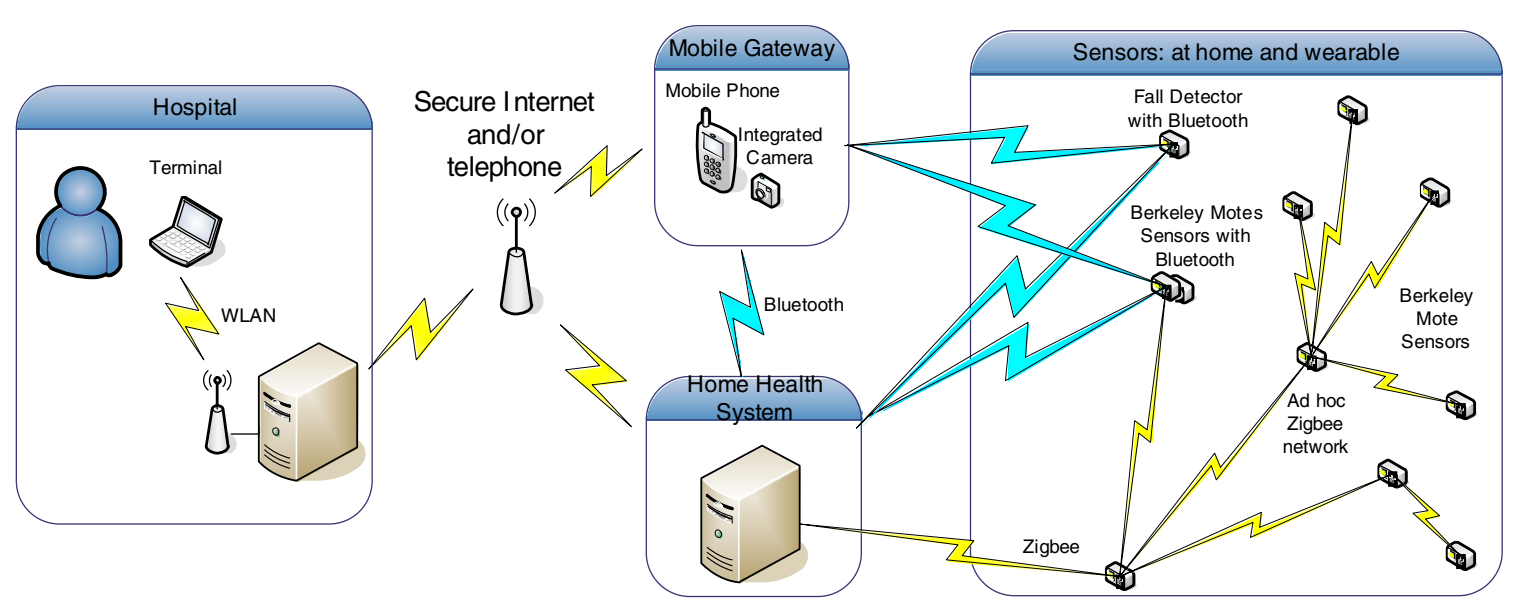

Fig. 1. SensorNet wireless connectivity in which each sensor device contains one or more functionalities.

In addition to providing alert notifications, the user can grant other people access to the user's health and status information, for example relatives or a health service provider that is able to remotely check and respond if the system detects something of concern regarding the user's health or activity, e.g. the blood pressure rises, a fall is detected, or other events of concern.

To enable this system, SensorNet provides a flexible, heterogeneous and secure wireless network that the sensor devices and event detection systems can communicate with.

\section{COMPONENTS}

The main components of SensorNet have been built to allow for development and further research into the devices and algorithms that will be part of the ITALH system. The construction of the SensorNet network is the first enabling step in the participatory design process for this project. With the basic system in place, sensor devices, protocols and algorithms can be developed through early prototyping, testing and evaluation, interaction with the users and stakeholders and iterative design. The components of SensorNet and how they integrate with the other components of ITALH are described here.

\section{A. Privacy}

An critical aspect about sensing data about people's health and activity is privacy. SensorNet and ITALH provides privacy in several ways that are inherent in the components of the system.

The sensor devices themselves contain embedded processors and software such that they will not transmit information about the user except to provide notification of an event of concern, or if specifically requested to do so by an authorized and authenticated user. While encryption can protect this data, it is much more secure to reduce the amount of data transmitted to a very low level. This also reduces the bandwidth requirement of the system. When data is streamed, for example when remote monitoring is authorized such that a care provider can check in on the user, then the signal will be encrypted for privacy.
This Home Health System is by default a personal and closed system. The person using the system can grant other people or services access to part of the data collected by the system. For example, the user of the system can grant (in advance) an emergency service access to cameras installed in the home and sensors worn by the user in the event that a fall is detected by the system, or the user can allow a doctor to access a user's medical data during an online appointment.

By letting the user explicitly decide who has access to the health and activity data, what data they have access to and in what kind of circumstances we begin to address the privacy issues.

\section{B. Sensors}

The project relies on the existence of ubiquitous and reliable sensors that are able to collect information about the wellbeing of the user of the system and their environment. Fixed wireless sensors are employed to monitor the environment and provide for an IEEE 802.11.4 (Zigbee) ad hoc wireless network (see section III-E below). This is done with Telos Rev B Mote devices [6] which provide built-in temperature, humidity and light sensors that allow for basic environmental sensing, and expansion for other sensing functionality. Wearable sensors are also included which measure and analyze the user's health and activity such as motion and, in the near future, heart rate.

The first wearable sensor application being integrated is that of the Ivy Project at Berkeley, which is an accelerometerbased fall sensing device [7]. In this project algorithms are being developed to detect falls and other motion-related events based on accelerometer data. These devices are worn on the belt and connect to SensorNet via Bluetooth to allow for direct connection to a standard Bluetooth-enabled, Symbian OS mobile telephone, such as the Nokia 6680 which is being used in the prototype.

Further home and bio-sensors will be connected as they become available and as determined by evaluation of the effectiveness of the current set and the need for further functionality. 


\section{Gateways}

SensorNet has two types of gateways: a mobile gateway that is portable and which the sensors worn by the user can send data to, and a fixed gateway used to connect sensors in the Home Health System.

The mobile gateway is a mobile telephone with Bluetooth connectivity and a programmable Symbian Operating System. The Nokia 6670 adn 6680 telephone are being used in the prototype. A secondary feature (for this application) of these phones is the integrated camera which provides the opportunity for two-way video conferencing. While the 6680 phone has $3 \mathrm{G}$ capability that in not available in the North America, video conferencing in the current prototype is enabled via the Bluetooth connection to the Home Health System and via the Internet to an authorized care giver, and this will be the primary video conferencing means in this project. The 6680 also provides significantly more processing power and a second camera to improve the video conferences capability.

The home gateway is provided by the Home Heath System which is a Windows XP based system. This has Bluetooth and Zigbee wireless connectivity provided by of-the-shelf Bluetooth devices and a USB connected Telos Rev B Mote device for Zigbee.

\section{Sensor Fusion and Network}

Alert notifications are sent from the sensors to the gateways which fuse the information and determine an appropriate response. For example, a belt-worn fall detection sensor might send an alert at which point the gateway might query a motion sensor in the appropriate room, and/or a pulse monitor to determine if an accident has occurred. If normal behavior is detected by the latter devices, then the event might just be recorded as an incident of interest, or the user might be prompted to ask if they are alright. If on the other hand no motion is detected then the gateway might immediately query the user and send an emergency signal if there is no response within a certain (short) period of time. With the emergency signal, access would be granted to the remote care provider who could $\log$ in and via the live camera data.

\section{E. Setup and User Interfaces}

Fundamental to the system is the ease of setup and use. The ad hoc nature of the wireless network makes it as easy and putting the home based sensors in places around the home and turning them on. Wearable sensors will require similar ease of use in many cases.

The home and mobile gateways will be programmed with various user interfaces suitable for users of varying ability (and interest), for caregivers, and for expert users such that the system can be maintained easily and interacted with very simply.

The participatory design process will also focus of the development of these user interfaces.

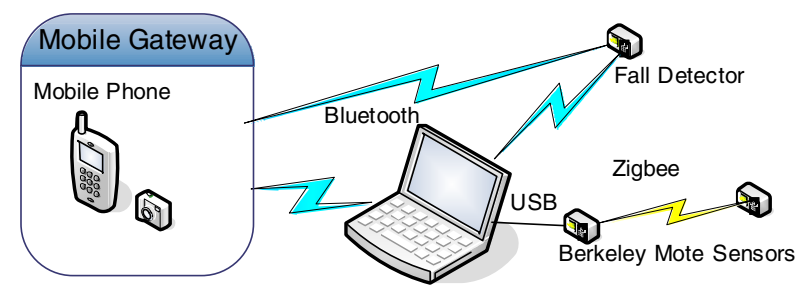

Fig. 2. SensorNet Development Setup

\section{RESUlTS}

The SensorNet system prototype currently connects a fall sensor to a mobile phone via a BlueGiga cable replacer module. The module phone streams the data from the sensor to a PC through Bluetooth and can also stream video from its cameras if requested by the system. To connect to the Zigbee, one mote is used as aUSB to Zigbee adapter as in Fig. 2. This emulates both the Home Heath System gateway and provides for development of the Bluetooth network protocols and easy programming and development of the Telos Motes. Embedded Bluetooth radios will be integrated into each sensor device after the sensor package hardware and embedded software has been throughly evaluated. The Telos Motes, however are already Zigbee enabled and have a well established development environment supporting the nesC programming language and TinyOS operating system [8], [9].

Additionally, connectivity between the mobile and home gateways exists to the remote caregiver station via a local ethernet network using a web browser interface. In the prototype system this interface is not yet a secure one, however. The home gateway currently receives streamed sensor data so that it can be used for analysis and algorithm development. The mobile gateway can stream video and pictures from the mobile phone camera to a home gate PC through a Bluetooth connection. It is also possible to remote control the system from the PC and decide when it is going to take pictures and start and stop the video stream.

\section{CONCLUSIONS AND FUTURE WORKS}

\section{A. Conclusions}

The wireless infrastructure of the Information Technology for Assisted Living at Home Project has been presented. SensorNet is a heterogenous wireless network using Bluetooth and Zigbee radios to connect a diverse set of embedded sensor devices. These devices and the network for a Home Health System which can monitor the health and activity of the use in a secure and private manner and issue alerts to the user, care givers or emergency services as necessary to provide additional safety and security to the user.

This system is being developed to provide this safety and security so that elder citizens who might have to leave their own homes for a group care facility will be able to extend their ability to remain at home longer. This will in most cases provide them with better quality of life and better health in a cost effective manner. 


\section{B. Future Works}

This project is being developed with participatory design between the users, care providers and developers. With the SensorNet infrastructure in place, sensor devices will be identified for development and implemented as the system is expanded in a modular manner to include a wide selection of devices.

The first testbed in which this is being tested is a retirement home in the San Francisco Bay Area. Follow these tests, the system will be shared with our partners in Denmark and Finland for further evaluation in group care facilities, hospitals and homes in all three regions.

\section{ACKNOWLEDGMENTS}

The authors gratefully acknowledge the assistance and participation of our ITALH partners Morten Kyng at University of Aarhus, Jari Hytinnen and Jukka Lekkala at Tampere University of Technology, and Ruzena Bajscy, Steve Glaser, Karric Kwong and Jay Chen of the Ivy Project at UC Berkeley and Jonathan Sprinkle at UC Berkeley for his hardware assistance.

\section{REFERENCES}

[1] M. V. M. Figueredo and J. S. Dias, "Mobile telemedicine system for home care and patient monitoring," in 26th Annual International Conference of the EMBS, September 2004.
[2] J. M. Choi, B. H. Choi, J. W. Seo, R. H. S. adn M S Ryu, W. Yi, and K. S. Park, "A system for ubiquitous health monitoring in the bedroom via a bluetooth network and wireless lan," in 26th Annual International Conference of the EMBS, September 2004.

[3] J. Rowan and E. D. Mynatt, "Digital family portrait field trial: Support for aging in place," in Proceedings of CHI, 2005, pp. 521-530.

[4] P. E. Ross, "Managing care through the air," IEEE Spectrum, pp. 26-31, December 2004.

[5] A. Sixsmith and N. Johnson, "Smart sensor to detect the falls of the elderly," IEEE Pervasive Computing, vol. 3, no. 2, pp. 42-47, AprilJune 2004.

[6] J. Polastre, R. Szewczyk, and D. Culler, "Telos: Enabling ultra-low power wireless research," in The Fourth International Conference on Information Processing in Sensor Networks: Special track on Platform Tools and Design Methods for Network Embedded Sensors (IPSN/SPOTS), April 25-27 2005.

[7] R. Bajcsy, J. Chen, K. Kwong, D. Chang, and J. Luk, "Fall detection using wireless sensor networks," in submitted to the 27th Annual International Conference of the EMBS, September 2005.

[8] D. Gay, P. Levis, R. von Behren, M. Welsh, E. Brewer, , and D. Culler, "The nesc language: A holistic approach to networked embedded systems," in Proceedings of Programming Language Design and Implementation (PLDI) 2003, June 2003.

[9] P. Levis, N. Lee, M. Welsh, and D. Cullerk, "Tossim: Accurate and scalable simulation of entire tinyos applications," in Proceedings of the First ACM Conference on Embedded Networked Sensor Systems (SenSys 2003), 2003. 